\title{
Structure-activity relationships of glutathione peroxidase 4 inhibitor warheads
}

\author{
John K. Eaton, ${ }^{1, *}$ Laura Furst, ${ }^{1}$ Luke L. Cai, ${ }^{1}$ Vasanthi S. Viswanathan, ${ }^{1, *}$ and Stuart L. \\ Schreiber ${ }^{1,2, *}$ \\ ${ }^{1}$ Broad Institute of MIT and Harvard, Cambridge, MA 02142, United States \\ 2 Department of Chemistry and Chemical Biology, Harvard University, Cambridge, MA 02138, United States
}

\section{Abstract:}

Direct inhibition of GPX4 requires covalent modification of the active-site selenocysteine. While phenotypic screening has revealed that activated alkyl chlorides and masked nitrile-oxides can inhibit GPX4 covalently, a systematic assessment of potential electrophilic warheads with the capacity to inhibit cellular GPX4 has been lacking. Here we survey more than 25 electrophilic warheads across several distinct GPX4-targeting scaffolds. Surprisingly, we find that electrophiles with attenuated reactivity compared to chloroacetamides are unable to target GPX4. The highly reactive propiolamide warheads we uncover in this study highlight the potential need for masking strategies similar to those we have described for nitrile-oxide-based GPX4 inhibitors. Finally, our observations that there are spatial requirements between warhead and scaffold for achieving optimal GPX4 targeting and that certain low-molecular-weight analogs inhibit GPX4 with selectivity suggest that rational design of GPX4 inhibitors may be a productive approach. The generation of ligand-bound crystal structures to facilitate such studies should therefore be prioritized by the field.

Keywords: covalent inhibitor, masked electrophile, prodrug, propiolamide, nitrile oxide, GPX4, ferroptosis

Glutathione peroxidase 4 (GPX4) is a selenocysteine-containing antioxidant protein that plays a critical role in protecting cells from ferroptosis, a non-apoptotic and iron-dependent form of cell death caused by lipid hydroperoxides. ${ }^{1-3}$ Induction of ferroptosis via inhibition of GPX4 has promising therapeutic potential, especially for targeting cancer cells that are otherwise therapy resistant. $^{4-6}$

However, GPX4 represents a difficult-to-drug target because it has a shallow active site that is not amenable to interacting with small molecules. ${ }^{7,8}$ This topology constitutes a unique feature among the broader family of glutathione peroxidases, enabling reduction of structurally-diverse lipid hydroperoxide substrates. The reliance on a nucleophilic selenocysteine residue and lack of a drug-like active site suggest that covalent inhibitors may be the most effective strategy to target GPX4 directly.

To date, all known cell-active GPX4 inhibitors inactivate GPX4 by forming a covalent bond with its catalytic selenocysteine residue. The vast majority of these inhibitors are activated alkyl chlorides such as chloroacetamides (Figure 1), ${ }^{2}$ which lack drug-like properties and have therefore not achieved widespread success in drug development. ${ }^{9,10}$ We recently identified masked nitrile oxides, including 4-nitroisoxazole ML210 (Figure 1 ) $^{10}$ and diacylfuroxans, ${ }^{11}$ as promising prodrugs for inhibiting GPX4 with selectivity. Nevertheless, the development of improved inhibitors will benefit from the discovery of novel GPX4-targeting warheads.

The narrow spectrum of electrophiles capable of binding GPX4 is a result of the failure to identify additional chemotypes either from high-throughput screens ${ }^{12-14}$ or structure-activity relationship (SAR) studies of (1S,3R)-RSL3 (referred to as RSL3), ${ }^{2,10,15}$ the first described GPX4 inhibitor. ${ }^{2}$ Here we attempt to address the lack of GPX4-targeting warheads by performing further systematic SAR studies. We investigate multiple known inhibitor scaffolds in combination with 
several warheads including haloacetamides possessing attenuated reactivity, ${ }^{16}$ acrylamides that feature in recent FDA-approved therapeutics (e.g., ibrutinib), and other electrophilic groups that have not been investigated previously in the context of GPX4 inhibitors.

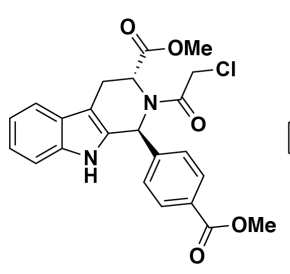

RSL3

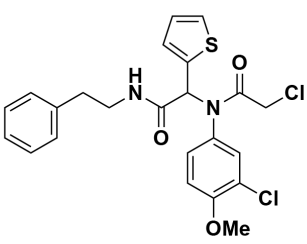

ML162

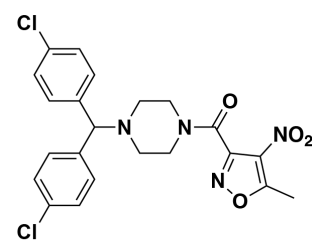

ML210

Figure 1: Small-molecule inhibitors of GPX4.

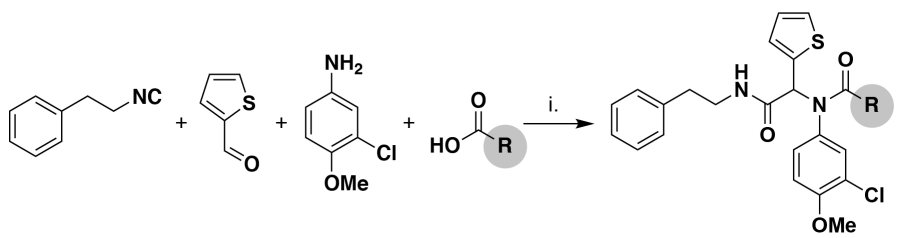

Scheme 1: Synthesis of ML162 analogs via Ugi 4-component reactions. i.) $\mathrm{MeOH}, 20{ }^{\circ} \mathrm{C}, 2-16 \mathrm{~h}, 39-87 \%$ yield

Our initial SAR studies focused on the chloroacetamide ML162, which is among the most potent GPX4 inhibitors. ${ }^{2,13}$ While preliminary SAR studies of ML162 indicate the importance of the chloroacetamide group, substitution with alternate electrophilic warheads has not been assessed previously. We therefore synthesized a collection of ML162 analogs (Table 1) using an Ugi fourcomponent reaction protocol. ${ }^{13}$ The ability of these analogs to kill cells via ferroptosis was then assessed in human LOX-IMVI melanoma cells. By measuring cell viability both in the absence and presence of ferrostatin-1 (fer-1), a lipophilic radical-trapping antioxidant that inhibits ferroptosis, ${ }^{1,17,18}$ we were able to determine the degree to which compounds selectively induce cell death via ferroptosis versus other mechanisms (e.g., apoptosis).

We found that none of the ML162 analogs exhibited improved potency (Table 1). Interestingly, analog 1 , which contains the more intrinsically reactive $\alpha$-bromoacetamide warhead, retained the ability to induce ferroptosis but with diminished potency and selectivity compared to ML162. We found that the chloroacetamide group cannot be replaced with other less-reactive electrophiles (2-9). These include $\alpha$-chlorofluoroacetamide (2), other haloacetamides (3-6), and acrylamide (7). However, we observed that the propiolamide analog 8 exhibited potent ferroptosis induction with improved selectivity compared to ML162. Similarly, a propiolamide group was the only electrophile able to replace the chloroacetamide warhead in RSL3 while retaining the ability to induce ferroptosis. ${ }^{15}$ Previous observations indicate that propiolamides are more reactive towards thiol nucleophiles than chloroacetamides ${ }^{19}$ and likely present similar challenges regarding stability, pharmacokinetics, and proteome-wide selectivity.

Many of the reactivity-tuning strategies applied to chloroacetamides ${ }^{16}$ or acrylamides ${ }^{20}$ are not chemically feasible for propiolamides. However, sterically hindered propiolamides, in a manner analogous to chloroacetamides ${ }^{9}$ and acrylamides, ${ }^{19}$ exhibit diminished reactivity toward nucleophiles. ${ }^{19}$ An attempt to attenuate the reactivity of the propiolamide warhead via incorporation of a terminal methyl group (9) resulted in complete loss of activity (Table 1), suggesting that it may not be possible to tune the reactivity of this warhead class through steric effects. Inspired by our studies of masked nitrile-oxide prodrugs for targeting GPX4, ${ }^{10,11}$ we hypothesized that it may be possible to mask the reactivity of the propiolamide warhead using a 
terminal protecting group that could be removed in situ and enable covalent inhibition of GPX4 in cells.

Table 1: Electrophilic warhead SAR of the ML162 scaffold.

(f)

a Values are determined from $n=4$ technical replicates ${ }^{\mathrm{b}}\left(\mathrm{EC}_{50}\right.$ with fer-1)/EC ${ }_{50}$

We therefore synthesized several analogs with protected propiolamide warheads (10-14, Table 2). Analogs bearing certain silyl protecting groups, including trimethylsilyl (11), triethylsilyl (12), and dimethylphenylsilyl (13), exhibit similar cellular activity to propiolamide 8 (Table 2). However, compound 14, which contains a more hindered triisopropylsilyl group, is unable to induce ferroptosis. We found that in phosphate-buffered saline solution (pH 7.4), 11 converts into 8 at room temperature within hours and forms identical covalent adducts with thiols (Supplementary Figure 1). In contrast, 14 is stable and does not react with thiols under identical conditions (Supplementary Figure 1). Our findings suggest the possibility of identifying a masking group possessing a suitable stability profile to enable delivery of propiolamide electrophiles in a prodrug form.

We next performed similar SAR studies of electrophilic warheads using the core benzhydrylpiperazine scaffold of ML210 without the nitroisoxazole group (15-31, Table 3). We found that only three of the tested compounds exhibit the ability to induce ferroptosis: chloroacetamide 15, propiolamide 16, and trimethylsilyl propiolamide 17. Tetrolamide 18 is inactive, as we observed for the corresponding ML162 analog 9 and related RSL3 analogs. ${ }^{15}$ Compared to both ML210 and other chloroacetamide- and propiolamide-based GPX4 inhibitors, 15-17 exhibit diminished potency and selectivity for ferroptosis induction. We confirmed that chloroacetamide 15 binds GPX4 in cells using the cellular thermal shift assay (CETSA) ${ }^{21}$ as well as GPX4 affinity enrichment with an alkyne analog (Supplementary Figure 2). The fact that both chloroacetamide and propiolamide warheads enable GPX4 inhibition when attached to the benzhydrylpiperazine scaffold, in addition to the core scaffolds of both RSL3 and ML162, reinforces the privileged nature of these electrophiles for targeting GPX4.

SAR studies for both ML162 (Table 1) and RSL3 ${ }^{2,10}$ indicate that acrylamide warheads are unable to target GPX4 effectively, and we also found this result to be the case for acrylamidecontaining ML210 analogs (19-22, 24). In addition to the acrylamide and propiolamide analogs, we prepared a collection of electrophilic (hetero)arenes capable of nucleophilic aromatic substitution $\left(S_{N} A r\right)$ reactions (25-31). This latter collection was inspired by the identification of selenocysteine-specific probes and covalent inhibitors of other selenoproteins that utilize warheads capable of $S_{N} A r$ chemistry. ${ }^{22-24}$ However, none of these warheads are capable of ferroptosis induction in LOX-IMVI cells (Table 3 ). 
Table 2: Investigation of masked propiolamides.

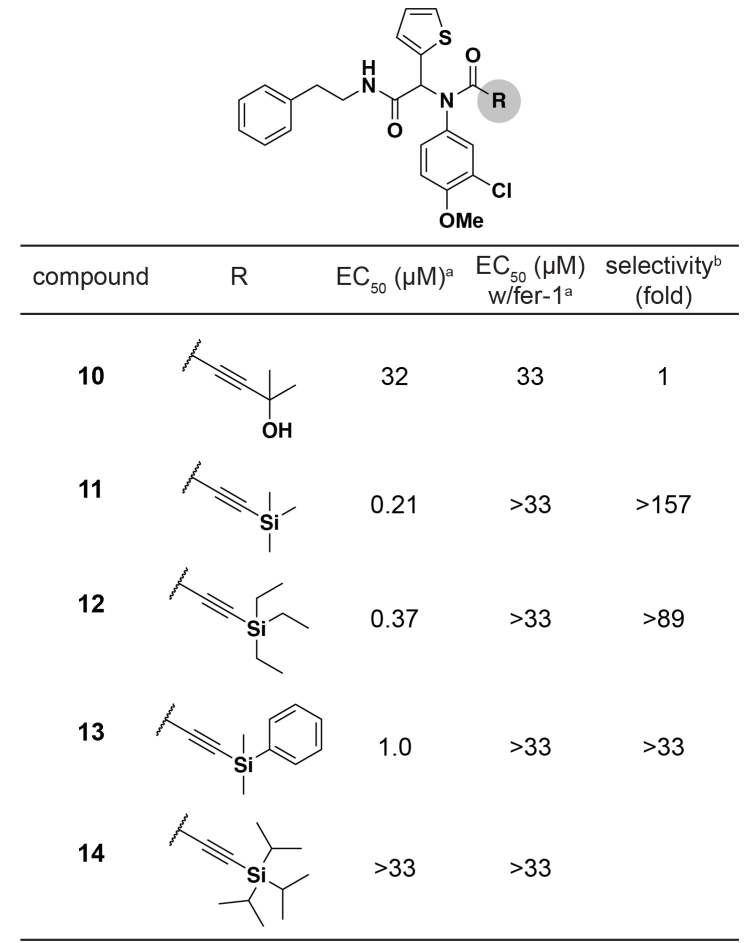

a Values are determined from $n=4$ technical replicates ${ }^{b}\left(\mathrm{EC}_{50}\right.$ with fer-1)/EC $\mathrm{E}_{50}$
Our ML162 and ML210 SAR studies reveal that only certain electrophiles, namely chloroacetamide and propiolamide, appear capable of direct GPX4 inhibition. This finding aligns with previous SAR studies featuring RSL3.,10,15 Our observations do, however, demonstrate that GPX4-targeting warheads and scaffolds are interchangeable in certain instances. On the basis of this discovery, we explored the activity of the nitroisoxazole warhead of ML210 in conjunction with the core scaffolds of several chloroacetamide-based GPX4 inhibitors (Table 4).

We prepared nitroisoxazole analogs of several chloroacetamide-based GPX4 inhibitors, including ML162, RSL3, DPI19, 2,25 and DPI13 ${ }^{2,25}$ (32-36, 38; Table 4). Assessment of these nitroisoxazoles revealed that most were unable to induce ferroptosis at the highest tested concentrations (Table 4), suggesting that this warhead is not generally able to facilitate covalent GPX4 inhibition. However, compound 35, the DPI19 scaffold functionalized with a nitroisoxazole warhead, is able to induce ferroptotic cell death (Table 4).

Cellular transformation of the nitroisoxazole group of ML210 into its active nitrile-oxide form requires two main steps: ring-opening hydrolysis of the nitroisoxazole and subsequent
dehydration of the resulting $\alpha$-nitroketoxime (Supplementary Figure $3 \mathrm{~A}$ ). ${ }^{10}$ It remains unclear whether enzymatic or purely chemical processes underlie these transformations, but our results indicate that there may be structural requirements distal to the nitroisoxazole that determine whether this prodrug mechanism is operative. We also note that we were unable to use standard hydrolysis conditions ${ }^{10,26}$ to convert certain nitroisoxazoles into their corresponding $\alpha$ nitroketoxime forms, including 32-34 (Supplementary Figures 3B,C). It may be the case that this initial activating transformation is also not achievable within live cells.

Another mechanistic hypothesis for inactive nitroisoxazole analogs is that the requisite transformations to the nitrile-oxide electrophile occur, but the resulting nitrile oxides are unable to target GPX4. One possibility may be that for certain inhibitor scaffolds, the nitrile-oxide group is not accessible to the selenocysteine residue for covalent modification. This caveat may explain why chloroacetamide (15) and propiolamide (16) analogs of ML210 exhibit diminished potency and selectivity for ferroptosis induction, as the electrophilic sites are at different positions in these compounds than they are in the active nitrile-oxide form of ML210.

To investigate this possibility, we synthesized an analog of ML162 wherein the chloroacetamide group is replaced with a nitrolic acid warhead (40, Figure 2$)$, which reacts with nucleophiles via a nitrile-oxide intermediate. ${ }^{10,27}$ Compound $\mathbf{4 0}$ induces ferroptotic cell death, but with diminished potency relative to ML162 and propiolamide analog $\mathbf{8}$. The different position of the electrophilic site of $\mathbf{4 0}$ likely contributes its lessened potency; the active nitrile-oxide species extends the position of the electrophilic site relative to the core scaffold by one carbon atom compared to the chloroacetamide in ML162 and changes the orientation relative to the propiolamide of $\mathbf{8}$. However, despite the diminished potency of $\mathbf{4 0}$, this result demonstrates that it is possible for an ML162 analog to target GPX4 through a nitrile-oxide electrophile. The electrophilic site in $\mathbf{4 0}$ is positioned similarly to the hypothetical site in $\mathbf{3 2}$ upon cellular activation 
of the nitroisoxazole. This parallel suggests that failure to unmask the nitrile-oxide electrophile may underlie the inactivity of $\mathbf{3 2}$ and possibly other nitroisoxazoles.

Table 3: Electrophilic warhead SAR with ML210 benzhydrylpiperazine scaffold

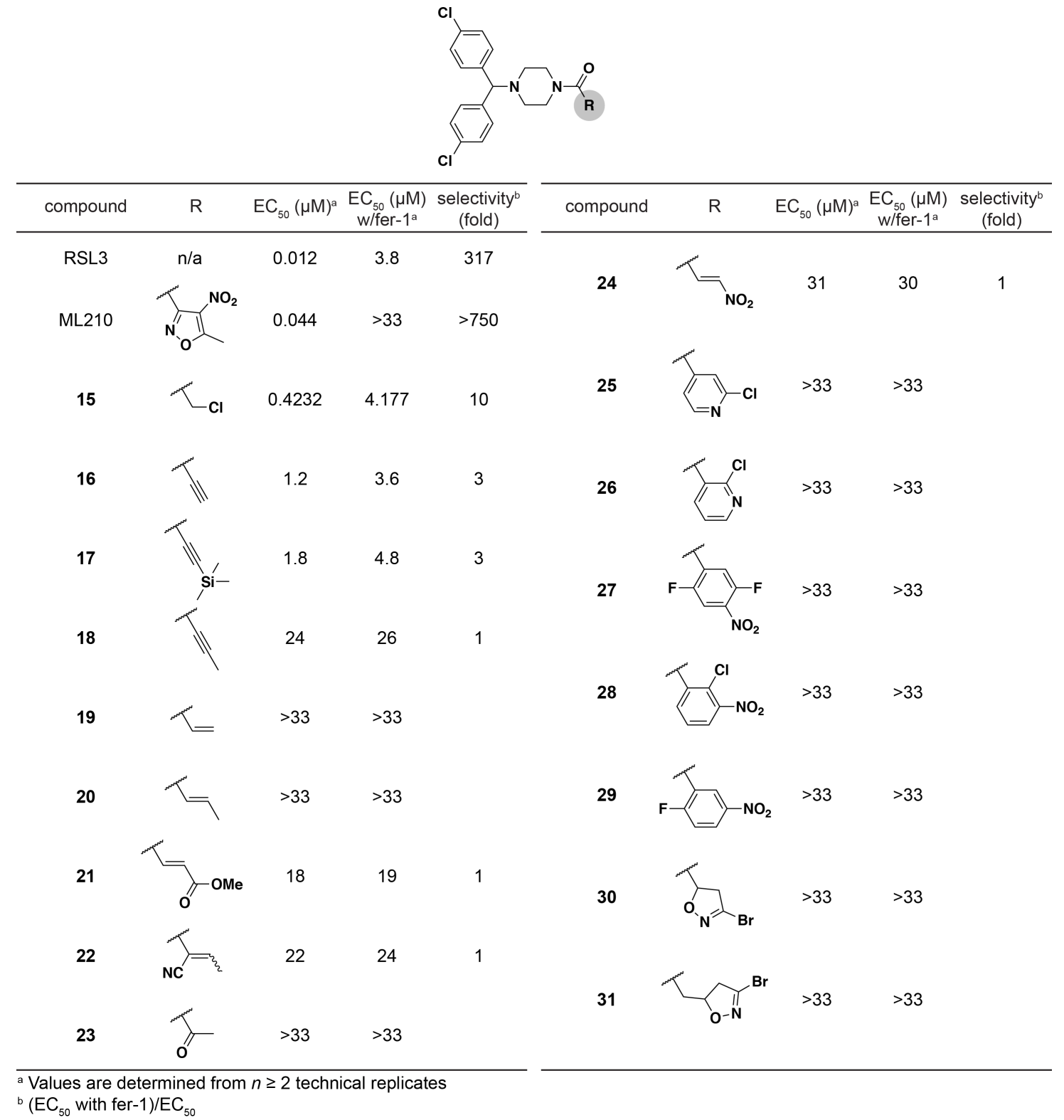

Our studies reveal that the nitroisoxazole warhead cannot necessarily substitute for other electrophiles in GPX4 inhibitors. This distinction is likely due to specific structural requirements for both multistep nitroisoxazole activation as well as for GPX4-targeting ability. However, we hypothesized that other structurally distinct nitroisoxazoles could target GPX4 without being appended to scaffolds of known GPX4 inhibitors. To understand the ability of nitroisoxazoles to target GPX4 more generally, we screened 30 nitroisoxazole-containing compounds in cell viability and fer-1 rescue experiments (Supplementary Table 1). Most lacked the ability to induce ferroptosis, but we identified several compounds inducing cell death that was rescued by fer-1 co-treatment. This collection includes two compounds that are close structural analogs of ML210, 
41 and 42 (Supplementary Table 1). In addition to these ML210-like compounds, we also observed two low-molecular-weight (low-MW) nitroisoxazoles, $\mathbf{4 3}$ and $\mathbf{4 4}$ (Figure 3A), that exhibit fer-1 rescuable cell killing (Figure 3B and Supplementary Table 1).

Table 4: Assessment of nitroisoxazole warheads on GPX4 inhibitor scaffolds

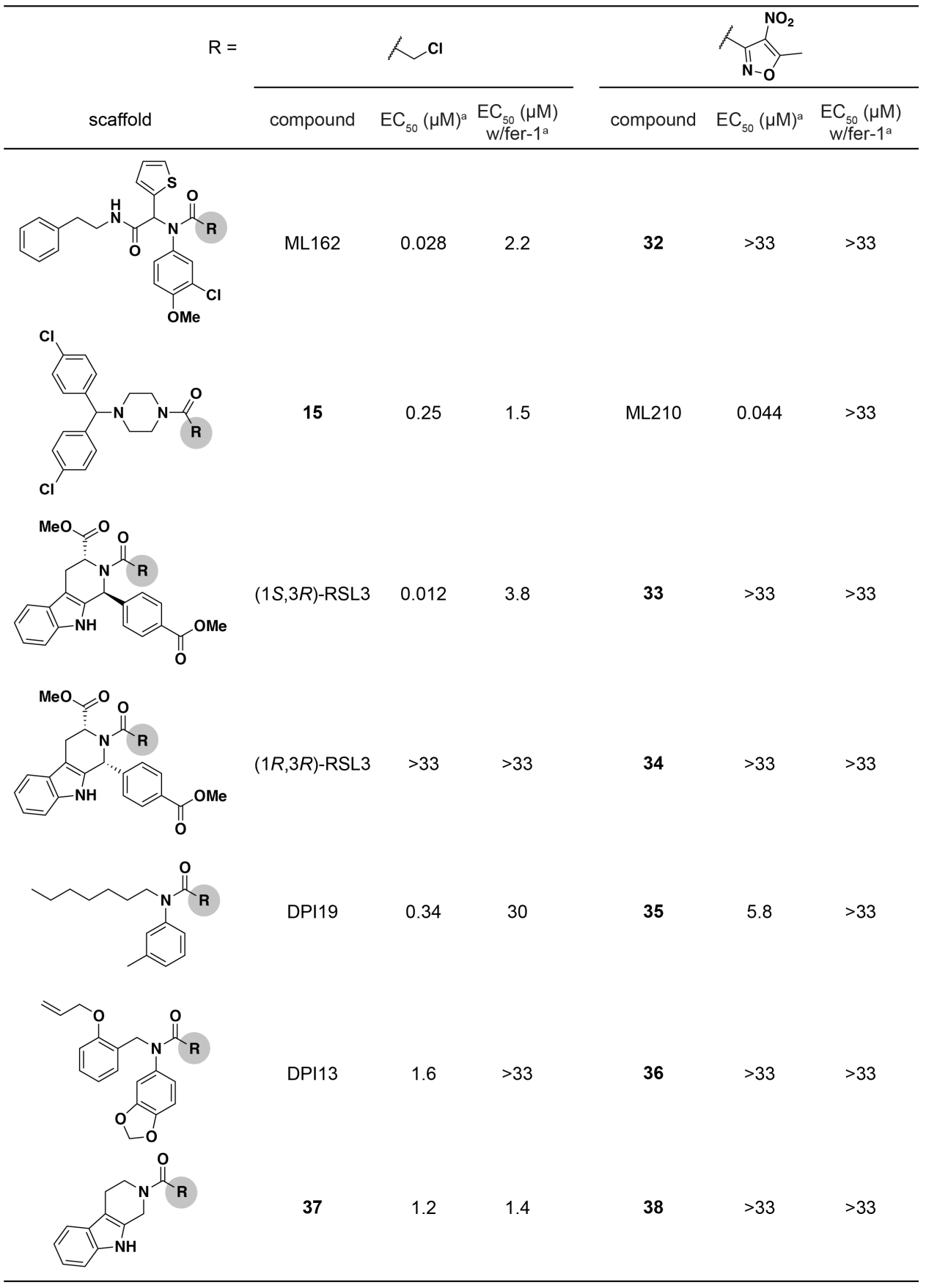

a Values are determined from $n \geq 2$ technical replicates 


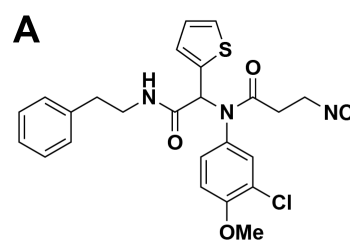

39

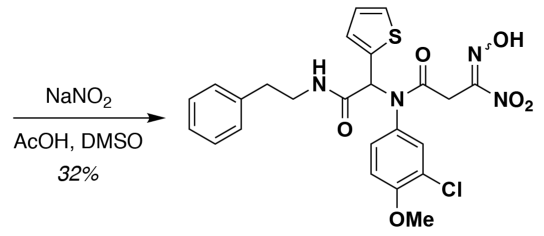

40
B

\begin{tabular}{ccc}
\hline compound & $\mathrm{EC}_{50}(\mu \mathrm{M})$ & $\begin{array}{c}\mathrm{EC}_{50}(\mu \mathrm{M}) \\
\text { w/fer-1 }\end{array}$ \\
\hline $\mathbf{3 9}$ & $>33$ & $>33$ \\
$\mathbf{4 0}$ & 10.1 & $>33$ \\
\hline
\end{tabular}

Figure 2: A nitrolic acid-containing analog of ML162 induces ferroptosis. (A) Synthesis of nitrolic acid 40. (B) The LOX-IMVI cell-killing activity of $\mathbf{4 0}$ can be suppressed by fer-1 co-treatment $(1.5 \mu \mathrm{M})$. Compound $\mathbf{3 9}$ does not affect cell viability at the concentrations tested. $\mathrm{EC}_{50}$ values were determined from four technical replicates.

Compound 44 contains a terminal alkyne group which can serve as a chemical handle for protein enrichment experiments (Figure 3A). We conjugated 44-labeled proteins to biotin-PEG3azide via $\mathrm{Cu}(\mathrm{I})$-catalyzed azide-alkyne cycloaddition (CuAAC) chemistry in order to assess the proteome-wide reactivity of this nitroisoxazole (Figure 3C). Enrichment of target proteins using solid-supported streptavidin beads enabled identification of the protein targets of this nitroisoxazole by SDS-PAGE and western blotting. We found that despite its small size, 44 exhibited surprising selectivity for a single major target protein of approximately $38 \mathrm{kDa}$ (Figure $3 \mathrm{C})$. The proteome-wide selectivity of $\mathbf{4 4}$ is reminiscent of recently reported low-MW isoxazolium probes based on Woodward's reagent K, which exhibit striking selectivity for macrophage migration inhibitor factor (MIF). ${ }^{28}$

Mass spectrometry-based proteomics revealed this protein to be glyceraldehyde 3-phosphate dehydrogenase (GAPDH), a protein we have previously found to be a major target of diacylfuroxans, another class of masked nitrile-oxide GPX4 inhibitors. ${ }^{11}$ A related nitroisoxazole probe, $\mathbf{4 5}$, is also able to bind GAPDH with similar selectivity compared to 44 while its nitro-free analog 46 does not (Supplementary Figures 4A,B). It is notable that, despite the structural similarity with $\mathbf{4 4}$, compound $\mathbf{4 5}$ does not induce ferroptosis and does not enrich GPX4 (Supplementary Table 1). We verified both GPX4 (Figure 3D) and GAPDH (Figure 3E) as covalent targets of $\mathbf{4 4}$ via western blotting experiments. As we reported previously, GPX4 was not detected by mass spectrometry-based proteomics, ${ }^{11}$ likely due to the low expression of GPX4 protein in LOX-IMVI cells. ${ }^{29}$ Compound $\mathbf{4 4}$ treatment also depletes GPX4 protein levels, as has been reported for many other ferroptosis-inducing compounds (Figure 3D and Supplementary Figure 4B), ${ }^{30-32}$ which further limits detection of GPX4 in proteomics experiments.

In this study, we explore the effectiveness of electrophilic warheads together with the core scaffolds of ML162, RSL3, and ML210. Our findings reveal that covalent GPX4 inhibitors must be highly electrophilic in order to react with the catalytic selenocysteine, a residue that is normally observed to exhibit greater nucleophilicity than cysteine. ${ }^{33}$ We speculate this requirement may be because the active-site environment of GPX4 suppresses the nucleophilicity of the selenocysteine residue, minimizing its reactivity towards electrophiles. Attenuation of the selenocysteine residue in this way provides a means for cellular systems to preserve the critical enzymatic function of GPX4 and prevent its alkylation by endogenous and environmental electrophilic species.

Due to the attenuated reactivity of the selenocysteine residue, we find that strategies used to modulate the reactivity of chloroacetamide ${ }^{9,16}$ warheads do not translate to GPX4 inhibitors. However, as we reported previously, ${ }^{10,11}$ prodrug electrophiles enable the use of highly reactive electrophiles to target GPX4. Here, we provide preliminary evidence that GPX4 inhibitors with propiolamide warheads can be similarly masked. We hope that these findings stimulate additional work to identify prodrug reactive groups to target GPX4 and other proteins.

Our studies also reveal that propiolamide, chloroacetamide, and nitroisoxazole groups are, in some instances, interchangeable as warheads for GPX4 inhibitors. As the position of the electrophilic site in each warhead is different, crystallographic studies will be necessary to understand the orientation of covalently-bound GPX4 inhibitors and identify noncovalent 
interactions important for potency and selectivity. Knowledge of how this diverse collection of inhibitors interacts with GPX4 will enable the rational design of improved GPX4-targeting compounds.

A<smiles>Cc1onc(C(=O)N(C)C)c1[N+](=O)[O-]</smiles>

43

B
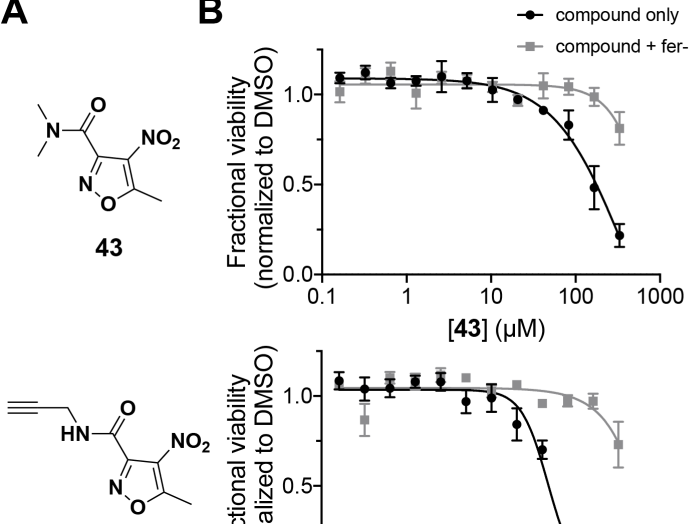

44

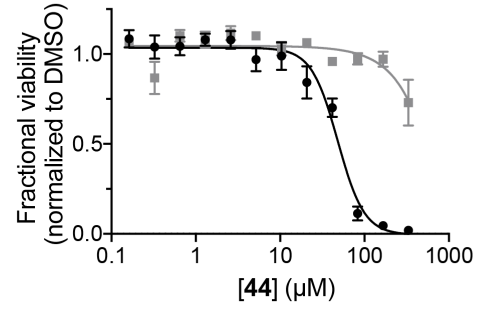

E

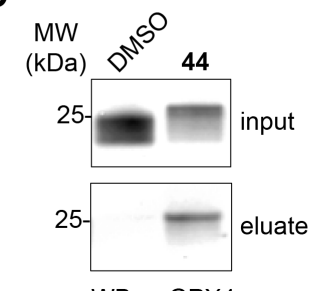

WB: $\alpha-G P X 4$

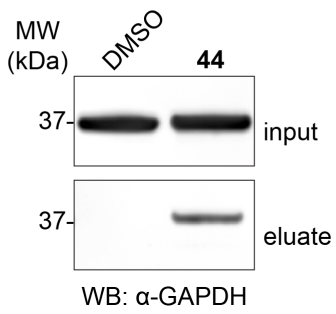

C

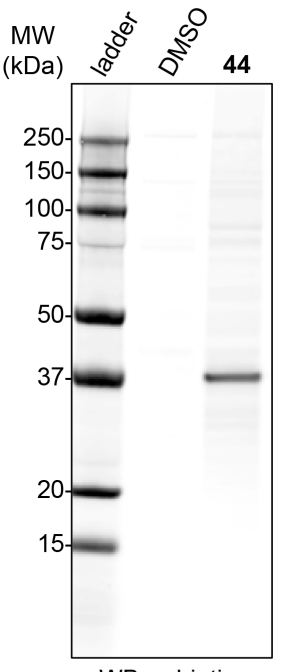

WB: a-biotin

Figure 3: Characterization of low-MW nitroisoxazole GPX4 inhibitors. (A) Chemical structures of $\mathbf{4 3}$ and $\mathbf{4 4}$. (B) 43 and $\mathbf{4 4}$ induce ferroptosis in LOX-IMVI cells. See also Supplementary Table 1. (C) Assessment of $\mathbf{4 4}(100 \mu \mathrm{M}, 16 \mathrm{~h})$ proteome-wide reactivity reveals labeling of a major target protein at $\sim 38 \mathrm{kDa}$. (D) Treatment of LOX-IMVI cells with $44(100 \mu \mathrm{M}, 16 \mathrm{~h})$ enables pulldown of GPX4. (E) Compound $\mathbf{4 4}$ covalently binds GAPDH.

\section{Author Information}

Corresponding Authors

* john.kittridge.eaton@gmail.com

* vasanthi.s.viswanathan@gmail.com

* stuart_schreiber@harvard.edu

ORCID iD

John K. Eaton: 0000-0003-4633-5546

Luke L. Cai: 0000-0003-4204-1938

Vasanthi S. Viswanathan: 0000-0001-9878-3629

Stuart L. Schreiber: 0000-0003-1922-7558

\section{Acknowledgements}

This work was supported by grants from the National Institutes of Health (R01GM038627 and R35GM127045). We thank Bogdan Budnik and Renee Robinson of the Harvard Center for Mass Spectrometry for assistance with proteomics. 


\section{Competing Interests}

S.L.S. serves on the Board of Directors of the Genomics Institute of the Novartis Research Foundation ("GNF"); is a shareholder and serves on the Board of Directors of Jnana Therapeutics; is a shareholder of Forma Therapeutics; is a shareholder and advises Kojin Therapeutics, Kisbee Therapeutics, Decibel Therapeutics and Eikonizo Therapeutics; serves on the Scientific Advisory Boards of Eisai Co., Ltd., Ono Pharma Foundation, Exo Therapeutics, and F-Prime Capital Partners; and is a Novartis Faculty Scholar.

\section{References}

(1) Dixon, S. J.; Lemberg, K. M.; Lamprecht, M. R.; Skouta, R.; Zaitsev, E. M.; Gleason, C. E.; Patel, D. N.; Bauer, A. J.; Cantley, A. M.; Yang, W. S.; et al. Ferroptosis: An IronDependent Form of Nonapoptotic Cell Death. Cell 2012, 149 (5), 1060-1072.

(2) Yang, W. S.; Sriramaratnam, R.; Welsch, M. E.; Shimada, K.; Skouta, R.; Viswanathan, V. S.; Cheah, J. H.; Clemons, P. A.; Shamji, A. F.; Clish, C. B.; et al. Regulation of Ferroptotic Cancer Cell Death by GPX4. Cell 2014, 156 (1-2), 317-331.

(3) Ingold, I.; Berndt, C.; Schmitt, S.; Doll, S.; Poschmann, G.; Buday, K.; Roveri, A.; Peng, X.; Porto Freitas, F.; Seibt, T.; et al. Selenium Utilization by GPX4 Is Required to Prevent Hydroperoxide-Induced Ferroptosis. Cell 2018, 172 (3), 409-422.e21.

(4) Viswanathan, V. S.; Ryan, M. J.; Dhruv, H. D.; Gill, S.; Eichhoff, O. M.; Seashore-Ludlow, B.; Kaffenberger, S. D.; Eaton, J. K.; Shimada, K.; Aguirre, A. J.; et al. Dependency of a Therapy-Resistant State of Cancer Cells on a Lipid Peroxidase Pathway. Nature 2017, 547 (7664), 453-457.

(5) Hangauer, M. J.; Viswanathan, V. S.; Ryan, M. J.; Bole, D.; Eaton, J. K.; Matov, A.; Galeas, J.; Dhruv, H. D.; Berens, M. E.; Schreiber, S. L.; et al. Drug-Tolerant Persister Cancer Cells Are Vulnerable to GPX4 Inhibition. Nature 2017, 551, 247-250.

(6) Tsoi, J.; Robert, L.; Paraiso, K.; Galvan, C.; Sheu, K. M.; Lay, J.; Wong, D. J. L.; Atefi, M.; Shirazi, R.; Wang, X.; et al. Multi-Stage Differentiation Defines Melanoma Subtypes with Differential Vulnerability to Drug-Induced Iron-Dependent Oxidative Stress. Cancer Cell 2018, 33 (5), 890-904.

(7) Scheerer, P.; Borchert, A.; Krauss, N.; Wessner, H.; Gerth, C.; Hohne, W.; Kuhn, H. Structural Basis for Catalytic Activity and Enzyme Polymerization of Phospholipid. Biochemistry 2007, 46 (31), 9041-9049.

(8) Borchert, A.; Kalms, J.; Roth, S. R.; Rademacher, M.; Schmidt, A.; Holzhutter, H. G.; Kuhn, H.; Scheerer, P. Crystal Structure and Functional Characterization of Selenocysteine-Containing Glutathione Peroxidase 4 Suggests an Alternative Mechanism of Peroxide Reduction. Biochim. Biophys. Acta - Mol. Cell Biol. Lipids 2018, 1863 (9), 1095-1107.

(9) Allimuthu, D.; Adams, D. J. 2-Chloropropionamide As a Low-Reactivity Electrophile for Irreversible Small-Molecule Probe Identification. ACS Chem. Biol. 2017, 12 (8), 21242131.

(10) Eaton, J. K.; Furst, L.; Ruberto, R. A.; Moosmayer, D.; Hilpmann, A.; Ryan, M. J.; Zimmermann, K.; Cai, L. L.; Niehues, M.; Badock, V.; et al. Selective Covalent Targeting of GPX4 Using Masked Nitrile-Oxide Electrophiles. Nat. Chem. Biol. 2020.

(11) Eaton, J. K.; Ruberto, R. A.; Kramm, A.; Viswanathan, V. S.; Schreiber, S. L. Diacylfuroxans Are Masked Nitrile Oxides That Inhibit GPX4 Covalently. J. Am. Chem. Soc. 2019, 141 (51), 20407-20415.

(12) Yang, W. S.; Stockwell, B. R. Synthetic Lethal Screening Identifies Compounds Activating Iron-Dependent, Nonapoptotic Cell Death in Oncogenic-RAS-Harboring Cancer Cells. Chem. Biol. 2008, 15 (3), 234-245.

(13) Weïwer, M.; Bittker, J. A.; Lewis, T. A.; Shimada, K.; Yang, W. S.; MacPherson, L.; 
Dandapani, S.; Palmer, M.; Stockwell, B. R.; Schreiber, S. L.; et al. Development of Small-Molecule Probes That Selectively Kill Cells Induced to Express Mutant RAS. Bioorganic Med. Chem. Lett. 2012, 22 (4), 1822-1826.

(14) Sakamoto, K.; Sogabe, S.; Kamada, Y.; Matsumoto, S.; Kadotani, A.; Sakamoto, J.; Tani, A. Discovery of GPX4 Inhibitory Peptides from Random Peptide T7 Phage Display and Subsequent Structural Analysis. Biochem. Biophys. Res. Commun. 2017, 482 (2), 195201.

(15) Jiang, C.; Chen, R.; Pandey, A.; Kalita, B.; Duraiswamy, A. J. US 2019/0263802 A1, 2019.

(16) Shindo, N.; Fuchida, H.; Sato, M.; Watari, K.; Shibata, T.; Kuwata, K.; Miura, C.; Okamoto, K.; Hatsuyama, Y.; Tokunaga, K.; et al. Selective and Reversible Modification of Kinase Cysteines with Chlorofluoroacetamides. Nat. Chem. Biol. 2019, 15 (3), 250258.

(17) Skouta, R.; Dixon, S. J.; Wang, J.; Dunn, D. E.; Orman, M.; Shimada, K.; Rosenberg, P. A.; Lo, D. C.; Weinberg, J. M.; Linkermann, A.; et al. Ferrostatins Inhibit Oxidative Lipid Damage and Cell Death in Diverse Disease Models. J. Am. Chem. Soc. 2014, 136 (12), 4551-4556.

(18) Zilka, O.; Shah, R.; Li, B.; Friedmann Angeli, J. P.; Griesser, M.; Conrad, M.; Pratt, D. A. On the Mechanism of Cytoprotection by Ferrostatin-1 and Liproxstatin-1 and the Role of Lipid Peroxidation in Ferroptotic Cell Death. ACS Cent. Sci. 2017, 3 (3), 232-243.

(19) Flanagan, M. E.; Abramite, J. A.; Anderson, D. P.; Aulabaugh, A.; Dahal, U. P.; Gilbert, A. M.; Li, C.; Montgomery, J.; Oppenheimer, S. R.; Ryder, T.; et al. Chemical and Computational Methods for the Characterization of Covalent Reactive Groups for the Prospective Design of Irreversible Inhibitors. J. Med. Chem. 2014, 57, 10072-10079.

(20) Bradshaw, J. M.; McFarland, J. M.; Paavilainen, V. O.; Bisconte, A.; Tam, D.; Phan, V. T.; Romanov, S.; Finkle, D.; Shu, J.; Patel, V.; et al. Prolonged and Tunable Residence Time Using Reversible Covalent Kinase Inhibitors. Nat. Chem. Biol. 2015, 11 (7), 525-531. Molina, D. M.; Jafari, R.; Ignatushchenko, M.; Seki, T.; Larsson, E. A.; Dan, C. Sreekumar, L.; Cao, Y.; Nordlund, P. Monitoring Drug Target Engagement in Cells and Tissues Using the Cellular Thermal Shift Assay. Science 2013, 341 (6141), 84-87. Maeda, H.; Katayama, K.; Matsuno, H.; Uno, T. 3'-(2,4-Dinitrobenzenesulfonyl)-2', 7'Dimethylfluorescein as a Fluorescent Probe for Selenols. Angew. Chemie Int. Ed. 2006, 45 (11), 1810-1813.

(23) Zhang, B.; Ge, C.; Yao, J.; Liu, Y.; Xie, H.; Fang, J. Selective Selenol Fluorescent Probes: Design, Synthesis, Structural Determinants, and Biological Applications. J. Am. Chem. Soc. 2015, 137 (2), 757-769.

(24) Stafford, W. C.; Peng, X.; Olofsson, M. H.; Zhang, X.; Luci, D. K.; Lu, L.; Cheng, Q.; Trésaugues, L.; Dexheimer, T. S.; Coussens, N. P.; et al. Irreversible Inhibition of Cytosolic Thioredoxin Reductase 1 as a Mechanistic Basis for Anticancer Therapy. Sci. Transl. Med. 2018, 10 (428), eaaf7444.

(25) Yang, W. S.; Kim, K. J.; Gaschler, M. M.; Patel, M.; Shchepinov, M. S.; Stockwell, B. R. Peroxidation of Polyunsaturated Fatty Acids by Lipoxygenases Drives Ferroptosis. Proc. Natl. Acad. Sci. 2016, 113 (34), E4966-E4975.

(26) Duranti, E.; Balsamini, C.; Spadoni, G.; Staccioli, L. Reaction of Secondary Acetylenic Bromides with Sodium Nitrite: Synthesis of 3,5-Alkyl(Aryl)-4-Nitroisoxazoles. J. Org. Chem. 1988, No. 53, 2870-2872.

(27) Egan, C.; Clery, M.; Hegarty, A. F.; Welch, A. J. Mechanism of Reaction of Isomeric Nitrolic Acids to Nitrile Oxides in Aqueous Solution. J. Chem. Soc. Perkin Trans. 2 1991, 249-256.

(28) Qian, Y.; Schürmann, M.; Janning, P.; Hedberg, C.; Waldmann, H. Activity-Based Proteome Profiling Probes Based on Woodward's Reagent K with Distinct Target 
Selectivity. Angew. Chemie - Int. Ed. 2016, 55 (27), 7766-7771.

(29) Doll, S.; Freitas, F. P.; Shah, R.; Aldrovandi, M.; da Silva, M. C.; Ingold, I.; Grocin, A. G.; Xavier da Silva, T. N.; Panzilius, E.; Scheel, C. H.; et al. FSP1 Is a GlutathioneIndependent Ferroptosis Suppressor. Nature 2019, 575 (7784), 693-698.

(30) Zhu, S.; Zhang, Q.; Sun, X.; Zeh, H. J.; Lotze, M. T.; Kang, R.; Tang, D. HSPA5 Regulates Ferroptotic Cell Death in Cancer Cells. Cancer Res. 2017, 77 (8), 2064-2077.

(31) Kagan, V. E.; Mao, G.; Qu, F.; Angeli, J. P. F.; Doll, S.; Croix, C. S.; Dar, H. H.; Liu, B.; Tyurin, V. A.; Ritov, V. B.; et al. Oxidized Arachidonic and Adrenic PEs Navigate Cells to Ferroptosis. Nat. Chem. Biol. 2017, 13 (1), 81-90.

(32) Shimada, K.; Skouta, R.; Kaplan, A.; Yang, W. S.; Hayano, M.; Dixon, S. J.; Brown, L. M.; Valenzuela, C. A.; Wolpaw, A. J.; Stockwell, B. R. Global Survey of Cell Death Mechanisms Reveals Metabolic Regulation of Ferroptosis. Nat. Chem. Biol. 2016, 12 (7), 497-503.

(33) Reich, H. J.; Hondal, R. J. Why Nature Chose Selenium. ACS Chem. Biol. 2016, 11 (4), 821-841. 